\title{
Out-of-equilibrium hydrodynamic fluctuations in the expanding QGP
}

\author{
Aleksas Mazeliauskas* \\ Institut für Theoretische Physik Universität Heidelberg 69120, Germany \\ E-mail: a.mazeliauskas@thphys.uni-heidelberg.de

\section{Yukinao Akamatsu} \\ Department of Physics, Osaka University, Toyonaka, Osaka 560-0043, Japan \\ E-mail: akamatsu@kern.phys.sci.osaka-u.ac.jp

\section{Derek Teaney} \\ Department of Physics and Astronomy, Stony Brook University, Stony Brook, New York 11794, \\ USA \\ E-mail: derek.teaneyestonybrook.edu
}

\begin{abstract}
We develop a set of kinetic equations for a correlator of thermal fluctuations which are equivalent to nonlinear hydro-dynamics with noise. We first show that the kinetic response precisely reproduces the one-loop renormalization of the shear viscosity for a static fluid previously discussed by Kovtun, Moore and Romatschke. We then use the hydro-kinetic equations to analyze thermal fluctuations for a Bjorken expansion. The rapid Bjorken expansion of a medium drives the hydrodynamic fluctuations out of equilibrium prescribed by the fluctuation-dissipation theorem. The steady state solution to the kinetic equations determine the coefficient of the first fractional power of the gradient expansion $\left(\propto 1 /(\tau T)^{3 / 2}\right)$, which was computed for the first time for Bjorken expansion. Away from the conformal limit, such non-linear noise corrections also induce a non-vanishing bulk viscosity. The formalism of hydro-kinetic equations can be applied to more general background flows, non-conformal systems and coupled to existing viscous hydrodynamic codes to incorporate the physics of hydrodynamic fluctuations, which become dominant near the critical point.
\end{abstract}

Critical Point and Onset of Deconfinement - CPOD2017

7-11 August, 2017

The Wang Center, Stony Brook University, Stony Brook, NY

${ }^{*}$ Speaker. 


\section{Introduction}

The Quark-Gluon Plasma (QGP) created in heavy ion collisions can be remarkably well described by relativistic viscous hydrodynamics. The successful heavy-ion modelling requires studying event-by-event fluctuations of the initial state and then propagating these fluctuations using viscous hydrodynamic codes, which include second order terms in the gradient expansion $[3,4,5$, $6,7]$. However thermal fluctuations, which formally contribute at the intermediate order, are not typically included in heavy ion simulations, with some notable exceptions [8, 9, 10, 11, 12, 13, 14].

Thermal fluctuations are always present in a dissipative system as dictated by the fluctuation dissipation theorem. The same microscopic degrees of freedom responsible for energy loss are exciting macroscopic-hydrodynamic fluctuations in the system. This results in a non-vanishing two point correlation functions of hydrodynamic fields, e.g. for momentum density in thermal equilibrium $g^{i}=T^{0 i}[15,16]$

$$
N_{g g}(t, k) \equiv\left\langle g^{i}(t, k) g^{j *}(t, k)\right\rangle=(e+p) T \delta^{i j} .
$$

In expanding system, the two point-correlation functions are no longer in equilibrium and one needs to calculate the out-of-equilibrium evolution of noise correlators. Such statistical fluctuations are potentially important for small systems like heavy ion collisions, where the number of constituents are order of thousands to tens of thousands. Dominance of stochastic fluctuations is also a prominent feature of the QCD critical point, which is the focus of the beam energy scan search at RHIC [20]. Therefore understanding the effects of perturbative hydrodynamic fluctuations in current heavy-ion simulations is an important step for studies of stochastic fluctuations close to the critical point.

In this work we present the effective kinetic description of hydrodynamic fluctuations $[1,2]$. First we determine the scale $k_{*}$ at which out-of-equilibrium hydrodynamic fluctuations dominate and then we proceed to derive the effective hydro-kinetic equations for the two point correlators of noise. We find the steady state solution of hydro-kinetic equations for the phenomenologically interesting case of Bjorken expansion and we compute the modification of the expectation of the energy-momentum tensor caused by the non-linear noise contributions. Finally, we apply this framework to non-conformal systems and we find the universal corrections to the QCD bulk viscosity.

\section{Separation of scales and hydro-kinetic equations}

The effective kinetic description of hydrodynamic fluctuations is based on the separation of scales of long wavelength hydrodynamic modes (which are never in thermal equilibrium and are determined by initial conditions), and short (but still macroscopic) hydrodynamic modes, which are damped and excited at the comparable rate to the background expansion.

In a dissipative system the modes with wavenumber $k$ are damped and excited at the rate proportional to viscosity $\sim \eta k^{2}$. In Bjorken expanding system, the external driving is given by the expansion rate $\partial_{\mu} u^{\mu}=1 / \tau$. These two rates are approximately equal at $k \sim k_{*}$, where

$$
k_{*} \sim \frac{1}{\sqrt{\gamma_{\eta} \tau}} \sim \frac{1}{c_{s} \tau \sqrt{\varepsilon}} .
$$




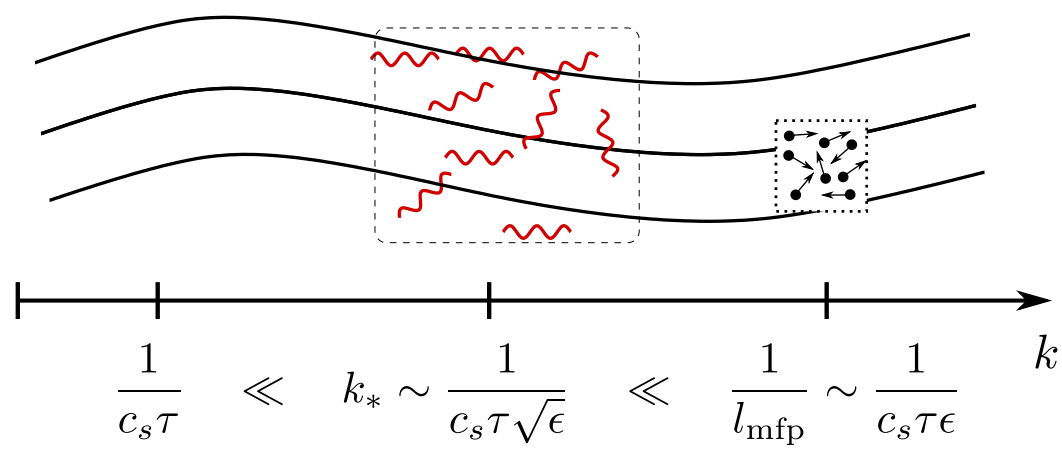

Figure 1: The separation of long-wavelength hydrodynamic modes $1 /\left(c_{s} \tau\right)$, the dominant out-ofequilibrium hydrodynamic modes $k_{*} \sim 1 /\left(c_{s} \tau \sqrt{\varepsilon}\right)$ and the microscopic modes $1 / l_{\mathrm{mfp}} \sim 1 /\left(c_{s} \tau \varepsilon\right)$, where $l_{\mathrm{mfp}}$ is the mean free path in the system, $1 / \tau$ the Bjorken expansion rate and $\varepsilon=l_{\mathrm{mfp}} /\left(c_{s} \tau\right) \ll 1$ is the hydrodynamic gradient expansion parameter.

Here $\varepsilon$ stands for the hydrodynamic expansion parameter, which is equal to the ratio between microscopic relaxation time (characterized by the mean free path $l_{\mathrm{mfp}}$ ) and the system expansion. The hydrodynamic expansion is valid for $\varepsilon=l_{\mathrm{mfp}} /(c \tau) \ll 1$, therefore $k_{*}$ is parametrically separated from the long hydrodynamic modes and the microscopic scales as shown in Fig. 1. Wavenumbers much higher than the scale $k \gg k_{*}$ remain in thermal equilibrium, while the long wavelength modes $k \ll k_{*}$ are phase space suppressed. Therefore $k_{*}$ represents the scale of the dominant outof-equilibrium hydrodynamic fluctuations.

The equation of motion in relativistic hydrodynamics with noise is given by the conservation of the energy-momentum tensor

$$
D_{\mu} T^{\mu v}=0
$$

and constitutive equations, which can be written as a sum $T^{\mu v}=T_{\text {ideal }}^{\mu v}+T_{\text {visc. }}^{\mu v}+S^{\mu v}$ of ideal, viscous and a stochastic noise term $S^{\mu v}$ with non-zero two-point expectation value $\left\langle S^{\mu v} S^{\rho \sigma}\right\rangle[15,16,17]$. Linearising hydrodynamic fluctuations around homogeneous boost invariant background

$$
e=e_{0}+\delta e, \quad \vec{g}=0+\left(e_{0}+p_{0}\right) \vec{v}
$$

one can derive the Langevin type equation $[1,2]$

$$
D_{\mu} \delta T^{\mu v}=0 \Leftrightarrow-\dot{\phi}_{a}(\tau, \vec{k})=\underbrace{i \mathscr{L}_{a b} \phi_{b}}_{\text {ideal }}+\underbrace{\mathscr{D}_{a b} \phi_{b}}_{\text {viscous }}+\underbrace{\xi_{a}}_{\text {noise }}+\underbrace{\mathscr{P}_{a b}(\tau) \phi_{b}}_{\text {expansion }}
$$

for an euclidean 4-vector of perturbations

$$
\phi_{a}(\tau, \vec{k}) \equiv\left(c_{s} \delta e, \vec{g}\right) .
$$

Fluctuations $\phi_{a}$ can be decomposed into four components in terms of eigenvectors $\left(e_{A}\right)_{a}$ of the ideal evolution operator $\mathscr{L}_{a b}$. Those correspond to the two sound modes $\phi_{+}$and $\phi_{-}$, and two transverse diffusive modes $\phi_{T_{1}}$ and $\phi_{T_{2}}$ with corresponding eigenvalues $\lambda_{ \pm}= \pm c_{s} k$ and $\lambda_{T_{1}}=\lambda_{T_{2}}=0$. Then we can write the governing equations for the two point correlation function of modes $\phi_{A} \equiv \phi_{a}\left(e_{A}\right)_{a}$ in eigenvector basis with $A=+,-, T_{1}, T_{2}$. We define the correlator $N_{A B}$ as

$$
\left\langle\phi_{A}(t, \vec{k}) \phi_{B}\left(t,-\vec{k}^{\prime}\right)\right\rangle \equiv N_{A B}(t, \vec{k})(2 \pi)^{3} \delta\left(\vec{k}-\vec{k}^{\prime}\right) .
$$


and the resulting equations for $N_{A B}$ from Eq. 2.4 (for a conformal fluid) are

$$
\begin{aligned}
& \partial_{\tau} N_{ \pm \pm}=\overbrace{-\frac{4}{3} \gamma_{\eta} K^{2}\left[N_{ \pm \pm}-\frac{T\left(e_{0}+p_{0}\right)}{\tau}\right]}^{\text {equilibration }}-\overbrace{\frac{1}{\tau}\left(2+c_{s}^{2}+\cos ^{2} \theta_{K}\right) N_{ \pm \pm}}^{\text {expansion }}, \\
& \partial_{\tau} N_{T_{1} T_{1}}=-2 \gamma_{\eta} K^{2}\left[N_{T_{1} T_{1}}-\frac{T\left(e_{0}+p_{0}\right)}{\tau}\right]-\frac{2}{\tau} N_{T_{1} T_{1}}, \\
& \partial_{\tau} N_{T_{2} T_{2}}=-2 \gamma_{\eta} K^{2}\left[N_{T_{2} T_{2}}-\frac{T\left(e_{0}+p_{0}\right)}{\tau}\right]-\frac{2}{\tau}\left(1+\sin ^{2} \theta_{K}\right) N_{T_{2} T_{2}} .
\end{aligned}
$$

where $\gamma_{\eta}=\eta /\left(e_{0}+p_{0}\right)$ and we use the wavenumber vector $\vec{K} \equiv\left(k_{x}, k_{y}, k_{\eta} / \tau\right)$ in spherical coordinates $\hat{K} \equiv \vec{K} /|\vec{K}|=\left(\sin \theta_{K} \cos \varphi_{K}, \sin \theta_{K} \sin \varphi_{K}, \cos \theta_{K}\right)$. Only the diagonal terms of correlation matrix survive, because the off-diagonal terms of $N_{A B}$ either vanish by orthogonality or can be neglected due to rapidly oscillating phase in rotating wave approximation [1].

We see that the two point correlations are relaxing to the instantaneous thermal equilibrium value with the rate $\sim \gamma_{\eta} K^{2}$ and driven away from the equilibrium by the expansion term $\sim \frac{1}{\tau}$. For $|\vec{K}| \gg k_{*}$ the two point functions are close to thermal equilibrium and the deviations are well described by $1 /\left(\gamma_{\eta} K^{2} \tau\right)$ expansion, e.g. for $N_{++}$

$$
\frac{N_{++}(\tau, \vec{k})}{T\left(e_{0}+p_{0}\right) / \tau}=1+\frac{c_{s}^{2}-\cos ^{2} \theta_{K}}{\frac{4}{3} \gamma_{\eta} K^{2} \tau}+\ldots
$$

\section{Modification of energy momentum tensor}

In the presence of hydrodynamic noise, the effective long wavelength energy momentum tensor is modified by the contributions coming from the two-point correlatios of out-of-equilibrium noise at scale $k \sim k_{*}$. Specifically, the energy and longitudinal pressure are increased by the nonlinear contributions of momentum fluctuations $\vec{g}=\left(T^{\tau x}, T^{\tau y}, \tau T^{\tau \eta}\right)$

$$
\begin{array}{r}
\left\langle T^{\tau \tau}\right\rangle=e+\frac{\left\langle\vec{g}^{2}\right\rangle}{e+p}, \\
\left\langle T^{z z}\right\rangle=c_{s}^{2} e-\frac{4 \eta}{3 \tau}+\frac{\left\langle\left(g^{z}\right)^{2}\right\rangle}{e+p} .
\end{array}
$$

The non-linear noise expectation can be written as an integral over the phase space of hydrodynamic modes [1], e.g.

$$
\left\langle\left(g^{z}\right)^{2}\right\rangle=\tau \int \frac{d^{3} K}{(2 \pi)}\left[\frac{N_{++}+N_{--}}{2} \cos ^{2} \theta_{K}+N_{T_{2} T_{2}} \sin ^{2} \theta_{K}\right] .
$$

The integral is divergent due to the equilibrium expectation value of $N_{A B}$ and the leading large $K^{2}$ expansion term, see Eq. 2.10. Regulating the integral by a UV cutoff $\Lambda$, the universal divergent terms can be computed explicitly and agrees with previous computations using diagrammatic approaches [18]. The divergent contributions reflect the fact that the initial bare pressure and viscosity are also cut-off dependent, but their sum is independent of $\Lambda$.

$$
\left\langle T^{z z}\right\rangle=\underbrace{p_{0}(\Lambda)+\frac{\Lambda^{3} T}{6 \pi^{2}}}_{\equiv p_{\text {phys }}}-\frac{4}{3 \tau} \underbrace{\left[\eta_{0}(\Lambda)+\frac{17 \Lambda T}{120 \pi^{2}} \frac{e_{0}+p_{0}}{\eta_{0}}\right]}_{\equiv \eta_{\text {phys }}}+\text { finite. }
$$


After absorbing divergent terms in the physical pressure and viscosity, the remaining finite term for longitudinal pressure is

$$
\frac{\left\langle T^{z z}(\tau)\right\rangle}{e+p}=\frac{p}{e+p}-\frac{4 \gamma_{\eta}}{3 \tau}+\frac{1.08318}{s\left(4 \pi \gamma_{\eta} \tau\right)^{3 / 2}}
$$

The finite correction (also known as long time tail) comes with the characteristic fractional power, which can be understood from the simple estimate of the phase space of modes around the critical scale $k_{*}$ and the equipartition of energy $\frac{1}{2} k_{\mathrm{B}} T$

$$
\left\langle T^{z z}\right\rangle_{\text {fluct. }} \sim \underbrace{\frac{1}{2} k_{B} T}_{\text {equipart \# of modes }} \underbrace{\int_{*}^{k_{*}} d^{3} k} \sim T k_{*}^{3} \sim T\left(\frac{1}{\gamma_{\eta} \tau}\right)^{3 / 2}
$$

The numerical factor in front of the fractional power is expansion specific. We found the steady state solutions of Eqs. 2.7, 2.8 and 2.9, and calculated the precise coefficient of the long-time tail for the Bjorken expanding background [1]. A realistic estimate of the long time tail contributions in heavy ion collisions shows that it is comparable in size to the second order gradient terms, which are usually included in hydrodynamic simulations [1]. Therefore the inclusion of hydrodynamic noise contributions is necessary for the faithful description of dynamics at second order in gradients.

In addition to the long time tail corrections, the energy and transverse pressure acquire contributions sensitive to transverse momentum fluctuations at initial times [1].

\section{Noise induced bulk viscosity}

The QCD equations of state obtained from lattice simulations is not conformal and the realistic description of QGP fluid involves a non-vanishing bulk viscosity $\zeta$ and temperature dependent speed of sound $c_{s}(T)[19]$.

The hydro-kinetic equations can be equally well applied to non-conformal systems [2]. Similarly to the conformal case, we expect modifications to the transport coefficients due to hydrodynamic fluctuations. In particular, we are interested in the cut-off dependent, but universal corrections to the bulk viscosity $\zeta$, similar to those for shear viscosity $\eta$ (see Eq. 3.4). To find the bulk renormalization terms, it is enough to look at small perturbations around the equilibrium. Such perturbations can be conveniently introduced by applying a bulk metric perturbation

$$
d s^{2}=-d t^{2}+\left(\delta_{i j}+h_{i j}\right) d x^{i} d x^{j}, \quad h_{i j}(t)=h \delta_{i j} e^{-i \omega t} .
$$

Following the steps we did for conformal case, we arrive at the two point correlator equations similar to Eqs. 2.7, 2.8 and 2.9, but now the expansion rate is characterized by the time derivative of $h_{i j}(t)$ and there are additional terms due to bulk viscosity $\zeta$ and non-constant speed of sound $c_{s}(T)[2]$.

After calculating the non-linear noise corrections to the constitutive equations, we find that the divergent terms can be absorbed by the suitable definition of physical energy, pressure and transport coefficients. Specifically, the cut-off independent bulk viscosity $\zeta$ is given by

$$
\zeta=\zeta_{0}(\Lambda)+\frac{\Lambda T^{2}}{2 \pi^{2}}\left[\left(\frac{1}{3}+\frac{T}{2} \frac{d c_{s}^{2}}{d T}-c_{s}^{2}\right)^{2} \frac{s}{\zeta_{0}+\frac{4}{3} \eta_{0}}+\left(\frac{1}{3}-c_{s}^{2}\right)^{2} \frac{2 s}{\eta_{0}}\right] .
$$




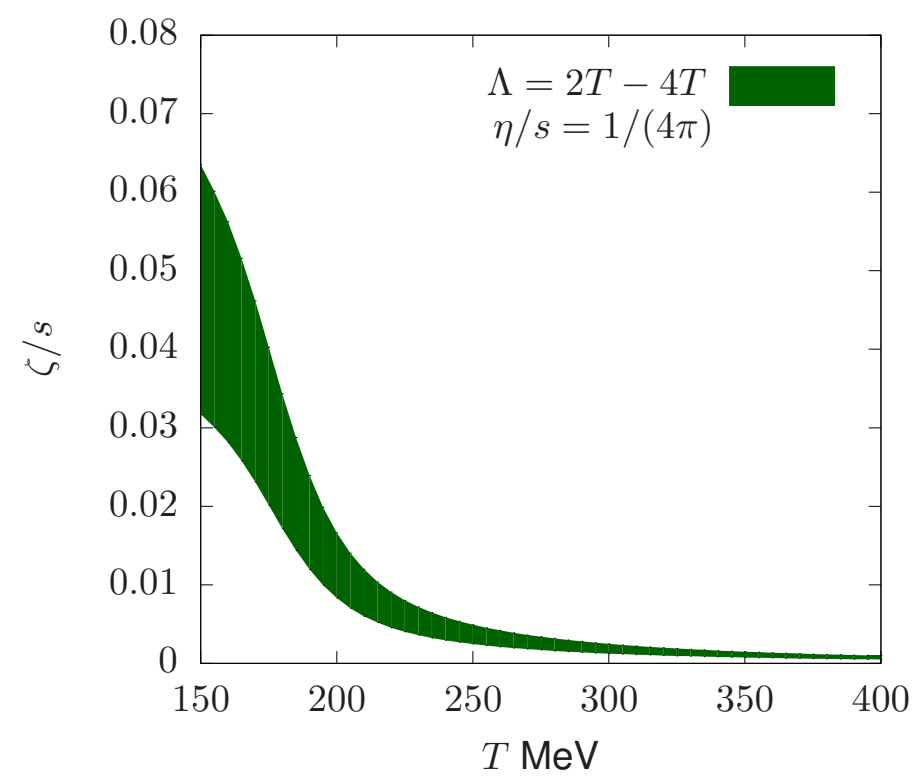

Figure 2: The QCD bulk viscosity induced by hydrodynamic noise fluctuations, Eq. 4.2, for $\eta / s=1 /(4 \pi)$ and a UV cut-off scale $\Lambda=2 T-4 T$. We use lattice QCD entropy density $s(T)$ and speed of sound $c_{s}(T)$, and neglect the bare viscosity $\zeta_{0}(\Lambda)[19]$

Note that the bulk renormalization depends on conformality breaking factors, e.g. $\left(\frac{1}{3}-c_{s}^{2}\right)^{2}$, and the shear viscosity $\eta_{0}$. The QCD transport coefficients are not easily calculable using lattice techniques, however the thermodynamic quantities like entropy density $s(T)$ and speed of sound $c_{s}(T)$ are well known [19]. The hydrodynamic modelling of heavy ion collisions indicates that the shear viscosity over entropy ratio is of order $\eta / s \sim 1 /(4 \pi)[3,5]$ and we can use Eq. 4.2 to estimate the QCD bulk viscosity due to hydrodynamic fluctuations for a UV cut-off $\Lambda=2 T-4 T$ of our theory. The result shown in Fig. 2 indicates a small, but conceptionally important contribution to bulk viscosity due non-equilibrium hydrodynamic fluctuations.

\section{Summary}

The presented framework of hydro-kinetic equations is a general and extendable way of calculating the physics of out-of-equilibrium noise in expanding systems. We successfully reproduced the universal renormalizations of bare energy, pressure and shear viscosity $\eta$ in agreement with previous diagrammatic calculations for conformal systems. We also calculated (for the first time) corrections to bulk viscosity $\zeta$ in non-conformal systems due to hydrodynamic fluctuations. We estimated the noise induced bulk viscosity for realistic parameters of QCD fluid.

The hydro-kinetic equations can be applied not only to systems close to equilibrium, but also in expanding backgrounds. We calculated the precise coefficient of fractional power corrections (or long time tails) due to out-of-equilibrium noise for the case of Bjorken expansion and found that it is comparable to second order gradients for typical system parameters in heavy ion collisions.

The hydro-kinetic equations is an alternative way of studying hydrodynamics with noise and can be profitably applied to a versatile range of hydrodynamic systems, e.g. with non-conformal equation of state or non-static backgrounds. 


\section{Acknowledgments}

Y.A.'s work at Stony Brook was supported through a JSPS Postdoctoral Fellowship for Research Abroad. A.M.'s and D.T.'s work was supported in part by the U.S. Department of Energy under Contract No. DE-FG02-88ER40388. A.M.'s work is supported in part by the DFG Collaborative Research Centre SFB 1225 (ISOQUANT).

\section{References}

[1] Y. Akamatsu, A. Mazeliauskas and D. Teaney, Phys. Rev. C 95 (2017) no.1, 014909 doi:10.1103/PhysRevC.95.014909 [arXiv:1606.07742 [nucl-th]].

[2] Y. Akamatsu, A. Mazeliauskas and D. Teaney, arXiv:1708.05657 [nucl-th].

[3] U. Heinz and R. Snellings, Ann. Rev. Nucl. Part. Sci. 63, 123 (2013) doi:10.1146/annurev-nucl-102212-170540 [arXiv:1301.2826 [nucl-th]].

[4] D. A. Teaney, doi:10.1142/9789814293297_0004 arXiv:0905.2433 [nucl-th].

[5] M. Luzum and H. Petersen, J. Phys. G 41, 063102 (2014) doi:10.1088/0954-3899/41/6/063102 [arXiv:1312.5503 [nucl-th]].

[6] C. Gale, S. Jeon and B. Schenke, Int. J. Mod. Phys. A 28, 1340011 (2013) doi:10.1142/S0217751X13400113 [arXiv:1301.5893 [nucl-th]].

[7] P. Romatschke, Int. J. Mod. Phys. E 19, 1 (2010) doi:10.1142/S0218301310014613 [arXiv:0902.3663 [hep-ph]].

[8] J. I. Kapusta, B. Muller and M. Stephanov, Phys. Rev. C 85, 054906 (2012) doi:10.1103/PhysRevC.85.054906 [arXiv:1112.6405 [nucl-th]].

[9] S. Gavin and M. Abdel-Aziz, Phys. Rev. Lett. 97, 162302 (2006) doi:10.1103/PhysRevLett.97.162302 [nucl-th/0606061].

[10] C. Young, J. I. Kapusta, C. Gale, S. Jeon and B. Schenke, Phys. Rev. C 91, no. 4, 044901 (2015) doi:10.1103/PhysRevC.91.044901 [arXiv:1407.1077 [nucl-th]].

[11] L. Yan and H. GrÃúnqvist, JHEP 1603, 121 (2016) doi:10.1007/JHEP03(2016)121 [arXiv:1511.07198 [nucl-th]].

[12] K. Murase and T. Hirano, Nucl. Phys. A 956, 276 (2016) doi:10.1016/j.nuclphysa.2016.01.011 [arXiv:1601.02260 [nucl-th]].

[13] K. Nagai, R. Kurita, K. Murase and T. Hirano, Nucl. Phys. A 956, 781 (2016) doi:10.1016/j.nuclphysa.2016.02.007 [arXiv:1602.00794 [nucl-th]].

[14] S. Gavin, G. Moschelli and C. Zin, Phys. Rev. C 94, no. 2, 024921 (2016) doi:10.1103/PhysRevC.94.024921 [arXiv:1606.02692 [nucl-th]].

[15] L.D. Landau and E.M. Lifshitz, Statistical Physics I, Course of theoretical physics, Pergamon Press (1980)

[16] E.M. Lifshitz and L.P. Pitaevskii, Statistical Physics II, Course of theoretical physics, Pergamon Press (1980)

[17] P. Kovtun, J. Phys. A 45, 473001 (2012) doi:10.1088/1751-8113/45/47/473001 [arXiv:1205.5040 [hep-th]]. 
[18] P. Kovtun, G. D. Moore and P. Romatschke, Phys. Rev. D 84, 025006 (2011) doi:10.1103/PhysRevD.84.025006 [arXiv:1104.1586 [hep-ph]].

[19] S. Borsanyi et al., Nature 539, no. 7627, 69 (2016) doi:10.1038/nature20115 [arXiv:1606.07494 [hep-lat]].

[20] X. Luo, Nucl. Phys. A 956, 75 (2016) doi:10.1016/j.nuclphysa.2016.03.025 [arXiv:1512.09215 [nucl-ex]]. 